\section{Chromogenic in situ hybridisation (CISH) should be an accepted method in the routine diagnostic evaluation of HER2 status in breast cancer}

HER2 protein overexpression or gene amplification is detected in approximately $20 \%$ of breast tumours, is associated with an aggressive phenotype and is predictive of response to trastuzumab therapy. The use of chromogenic in situ hybridisation (CISH) for evaluating HER2 status routinely is recognised in much of Europe but has yet to achieve widespread acceptance in the UK. CISH analysis for HER2 status was performed on 161 breast cancer cases. Results were compared with immunohistochemistry (IHC) and dual-colour fluorescence in situ hybridisation (FISH) data. There was $100 \%$ concordance between CISH and FISH, but only $93.8 \%$ concordance between CISH and IHC. Performing CISH 'in-house' was found to cost approximately $50 \%$ less than the FISH/IHC protocol at the reference laboratory. It is concluded that CISH is as accurate as FISH for diagnostic purposes and is more costeffective than the IHC/FISH regimen currently favoured in the UK.

Recent data show that amplification of the HER-2 gene is the most reliable predictor of response to trastuzumab therapy, ${ }^{2}$ indicating that a gene-based assay, rather than a protein overexpression assay, would be the most suitable type of analysis for HER2 status in breast tumour samples.

With the recent change in the licensing of trastuzumab to include its use as an adjuvant therapy (NICE 2006), it is important that patients most likely to benefit from its use are accurately identified. This change in the use of trastuzumab has increased the workload of histopathology laboratories significantly as well as creating an additional financial burden for hospital Trusts. We investigated the possibility of setting up HER2 analysis within our pathology department. Chromogenic in situ hybridisation (CISH), like fluorescence in situ hybridisation (FISH), directly visualises the number of gene copies present in the nucleus, it is cheaper and it produces a permanent record of the slide that can be interpreted with a light microscope in the context of the tumour histopathology. CISH and FISH have been compared for their sensitivity and specificity in numerous previous reports from across Europe, ${ }^{3-7}$ but CISH is not widely used in the UK. ${ }^{1}$ The current study was done as a validation study prior to setting up a HER2 testing service using CISH, but it was felt that our experience may be of interest to other laboratories considering setting up their own HER2 testing service.

\section{Methods}

One hundred and sixty-one breast cancer cases for which material was obtainable and that had immunohistochemistry (IHC) and/or FISH data available were chosen for the study. The samples had been analysed by the DAKO HercepTest IHC (DakoCytomation, Ely, Cambridgeshire, UK) and a small number of them $(n=24)$ had required confirmation by PathVysion dual-colour FISH (Abbott UK, Queensborough, Kent, UK) mostly because they were IHC $2+$. The manufacturer's protocols and scoring systems for both procedures were followed. The same cases were subsequently examined using a commercially available CISH assay (Zymed, Invitrogen, Paisley, UK), and the manufacturer's protocol was followed. The resulting slides were examined independently by two pathologists using light microscopy. The areas of invasive tumour were identified and the HER2 status was scored using the manufacturer's guidelines. Amplification was recorded when the nuclei of $>50 \%$ cells contained clusters, multiple dots $(>5)$ or a mixture of both. No amplification was recorded when the nuclei of $>50 \%$ cells contained one or two small dots. At least four areas of the tumour were examined to overcome issues of heterogeneity. Samples with 35 and 5-10 dots were further analysed using a chromosome 17 centromeric probe to confirm highly proliferating tumours in the former and cases of chromosome 17 numerical aberration in the latter. The cost implications for both the protocols were assessed.

\section{Results}

Results were obtained for all 161 samples tested. Amplified and non-amplified cases were readily distinguishable in the majority of cases (fig 1), whilst chromosome 17 correction was required in 19 cases $(10.6 \%)$ to confirm interpretation.

CISH and IHC showed $93.8 \%$ concordance (151/161) (table 1). Dual-colour FISH and CISH showed $100 \%$ concordance (table 1 ). There were seven $(4.4 \%)$ IHC $3+$ cases that were found by CISH to be not amplified. In each one of these seven cases, the results obtained with CISH were re-confirmed by dual-colour FISH analysis. One case $(0.6 \%)$, originally scored IHC $1+$, showed amplification by CISH, again re-confirmed by dual-colour FISH. There was $100 \%$ agreement between the two examining pathologists.

\section{Discussion}

FDA (Food and Drug Administration) approved HercepTest IHC analysis has been adopted as the frontline test for identifying patients eligible for trastuzumab therapy. A total of $5 \%$ of the samples in our series did not show the expected correspondence between HER2 gene status and HER2 protein expression, similar to previous reports. ${ }^{4}$ Our data indicate that $11 \%$ of women eligible for trastuzumab therapy on the basis of the IHC result are unlikely to benefit from it.

Misdirected therapy will potentially cost health authorities significant sums of money. A recent cost-effectiveness analysis for HER2 testing and trastuzumab therapy ${ }^{9}$ concluded that it is more cost-effective to use FISH alone or as confirmation of all IHC $2+$ and $3+$ results, rather than the current system. Whilst an expanded two-tier analysis will address the issue of false positivity, it will not identify those IHC-negative/FISH-positive cases that we and others have found in a proportion of tumours $(0.6-4.4 \%){ }^{10}$ Therefore, a frontline ISH test would be a more favourable option. Dual-colour FISH is accepted as the gold standard for HER2 analysis; CISH has been shown here and previously ${ }^{51-13}$ to be as sensitive and specific. Other reports have shown a high but variable level of concordance $(93.8-96 \%) .^{3614}$ The small number of discordant results in these reports have frequently

Table 1 HER2 IHC and FISH versus $\mathrm{CISH}$ data

\begin{tabular}{|c|c|c|c|c|c|c|c|c|}
\hline & \multicolumn{4}{|c|}{ IHC } & & \multicolumn{3}{|c|}{ FISH } \\
\hline & & $3+$ & $2+$ & $0 / 1+$ & & & + & - \\
\hline $\mathrm{CISH}$ & $\begin{array}{l}+ \\
-\end{array}$ & $\begin{array}{l}29 \\
7\end{array}$ & $\begin{array}{l}4 \\
12\end{array}$ & $\begin{array}{l}1 \\
108\end{array}$ & $\mathrm{CISH}$ & $\begin{array}{l}+ \\
-\end{array}$ & $\begin{array}{l}5 \\
0\end{array}$ & $\begin{array}{l}0 \\
19\end{array}$ \\
\hline
\end{tabular}

$\mathrm{CISH}$, chromogenic in situ hybridisation; FISH, fluorescence in situ hybridisation; IHC, immunohistochemistry.

Figure 1 (A) Core biopsy of invasive ductal carcinoma. Cells have been hybridised with a HER2 gene probe and visualised using an anti-digoxigenin peroxidase antibody, developed with $3^{\prime}, 3^{\prime}$-diaminobenzidine. Chromogenic in situ hybridisation analysis shows large clusters of hybridised HER2 probe, indicating the presence of a high level of HER2 gene amplification. (B) Core biopsy of invasive ductal carcinoma. One or two dots can be seen in each nucleus, indicating the absence of HER2 gene amplification. (C) Core biopsy of invasive ductal carcinoma. Small clusters and multiple dots can be seen, indicating a low level amplification of the HER2 gene. The insert shows analysis using the probe for the chromosome 17 centromere which indicates absence of polysosmy. 


\section{Take-home messages}

- Use of an in situ hybridisation assay in place of immunohistochemsitry would identify more accurately those women who would benefit from trastuzumab therapy.

- Chromogenic in situ hybridisation (CISH) is as sensitive and as specific as fluorescence in situ hybridisation (FISH)

- $\mathrm{CISH}$ is more cost-effective than FISH

been in the borderline samples due to the slight difference in cut-off level between CISH and FISH. ${ }^{14}$ A HER2:CEP17 ratio of $>2$ is classed as amplified by FISH; however, with CISH (Zymed), a score of $>5$ HER2 signals is classed as amplified. A consensus should be reached on a clinically relevant cut-off value for amplification for both ISH protocols.

Other FISH/CISH discrepancies have been due to inadequate use of chromosome 17 correction and difficult histology. ${ }^{36}$ By using chromosome 17 correction in all low amplified cases, light microscopy for assessment of HER2 in the histological context of the tumour sample and having experienced histopathologists analysing all the slides, we have not encountered these discrepancies between FISH and CISH results leading us to believe that CISH can reliably be used to assess breast tumour samples for patient eligibility for trastuzumab therapy.

\section{Acknowledgements}

We would like to thank Roche Products Ltd for the funding received for this study. The authors are independent of the funding organisation which sought no control over the design or content of the study.

S Di Palma, N Collins, C Faulkes, B Ping, G Ferns, B Haagsma, G Layer, M W Kissin, M G Cook

Histopathology, Royal Surrey County Hospital, Egerton Road, Guildford, Surrey GU2 7XX, UK

Correspondence to: S. Di Palma, Histopathology, Royal Surrey County Hospital, Egerton Road, Guildford, Surrey GU2 7XX, UK; silvana.dipalma@royalsurrey.

nhs.uk

doi: $10.1136 /$ icp. 2006.043356

Accepted for publication 11 January 2007

Published Online First 5 February 2007

Competing interests: none declared.

\section{References}

1 Ellis IO, Bartlett J, Dowsett M, et al. Best Practice No 176: Updated recommendations for HER2 testing in the UK. J Clin Pathol 2004;57:233-7.

2 Slamon DJ, Leyland-Jones B, Shak S, et al. Use of chemotherapy plus a monoclonal antibody against HER2 for metastatic breast cancer that overexpresses HER2. N Engl J Med 2001;344:783-92.

3 Saez A, Andreu FA, Segui MA, et al. HER-2 gene amplification by chromogenic in situ hybridisation (CISH) compared with fluorescence in situ hybridisation (FISH) in breast cancer-a study of two hundred cases. Breast 2006; 15:519-27.

4 Pauletti G, Dandekar S, Rong H, et al. Assessment of methods for tissue-based detection of the HER-2/ neu alteration in human breast cancer: a direct comparison of fluorescence in situ hybridization and immunohistochemistry. J Clin Oncol 2000; 18:3651-64

5 Gong Y, Gilcrease M, Sneige N. Reliability of chromogenic in situ hybridization for detecting HER2 gene status in breast cancer: comparison with fluorescence in situ hybridization and assessment of interobserver reproducibility. Mod Pathol 2005;18:1015-21.

6 Isola J, Tanner M, Forsythe A, et al. Interlaboratory comparison of HER-2 oncogene amplification as detected by chromogenic and fluorescence in situ hybridization. Clin Cancer Res 2004;10:4793-8.

7 Laakso M, Tanner M, Isola J. Dual-colour chromogenic in situ hybridization for testing of HER2 oncogene amplification in archival breast tumours. J Pathol 2006;210:3-9.

8 Bartlett JM, Going JJ, Mallon EA, et al. Evaluating HER2 amplification and overexpression in breast cancer. J Pathol 2001;195:422-8.

9 Elkin EB, Weinstein MC, Winer EP, et al. HER-2 testing and trastuzumab therapy for metastatic breast cancer: a cost-effectiveness analysis. J Clin Oncol 2004;22:854-63.

10 Saver T, Wiedswang G, Boudjema G, et al. Assessment of HER-2/neu overexpression and/or gene amplification in breast carcinomas: should in situ hybridization be the method of choice? Apmis 2003; 111:444-50.

11 Bhargava R, Lal P, Chen B. Chromogenic in situ hybridization for the detection of HER-2/neu gene amplification in breast cancer with an emphasis on tumors with borderline and low-level amplification: does it measure up to fluorescence in situ hybridization? Am J Clin Pathol 2005; 123:237-43.

12 Hauser-Kronberger C, Dandachi N. Comparison of chromogenic in situ hybridization with other methodologies for HER2 status assessment in breast cancer. J Mol Histol 2004;35:647-53.

13 Dandachi N, Dietze O, Hauser-Kronberger C. Chromogenic in situ hybridization: a novel approach to a practical and sensitive method for the detection of HER2 oncogene in archival human breast carcinoma. Lab Invest 2002;82:1007-14.

14 Hanna WM, Kwok K. Chromogenic in-situ hybridization: a viable alternative to fluorescence in-situ hybridization in the HER2 testing algorithm. Mod Pathol 2006;19:481-7.

\section{Histological findings of avulsion tear-type medial patellofemoral ligament injury in acute patellar dislocation}

Acute patellar dislocation is a common injury that occurs during a variety of activities and accounts for $2-3 \%$ of knee injuries. ${ }^{1}$ Since 1990 , especially over the past 5 years, more attention has focused on the medial patellofemoral ligament (MPFL) of the medial retinacular components. In several biomechanical studies, the MPFL has proved to be the primary restraint to lateral translation of the patella among the medial patellar stabilisers, and many clinical findings have shown that acute patellar dislocation is associated with MPFL injuries at a high rate. ${ }^{2-9}$

In 1999, Nomura ${ }^{6}$ classified MPFL injury in acute patellar dislocation into two types, avulsion tear and substantial tear. An avulsion tear is a detachment-type injury limited to the undersurface of the MPFL at the femoral attachment. It was suspected that this injury may result from a specific structure of the MPFL at the femoral attachment.

This study was to evaluate the histological findings of the avulsion tear-type injury of the MPFL in acute patellar dislocation.

\section{Case report}

A 23-year-old woman sustained initial dislocation of the left knee while climbing a step (case
1). Five days after the injury, we performed open MPFL surgery. From macroscopic observation at the time of the operation, an avulsion tear-type injury of the MPFL was found. The MPFL seemed to be intact, but when the ligament was lifted, the avulsed site was found (fig 1A,B).

An 18-year-old woman was injured when someone bumped against her, forcing her right knee to apply valgus and rotation force (case 2 ). Eleven days after the injury, we performed open MPFL surgery. From macroscopic observation, the MPFL seemed to be intact. A massive clot was present under the femoral attachment, and an avulsion tear was diagnosed.

\section{Pathology}

In the two cases, we obtained the histological samples of the injured MPFL at or near the femoral attachment, and histological findings were examined. The sections were stained with H\&E.

The superficial layer of the MPFL in case 1 seemed almost intact. The hyalinised collagen bundles showed lamellar arrangement. In the deep layer, a granulation tissue response was encountered. The proliferation of capillaries and fibroblasts with a mild degree of infiltration of lymphocytes and histiocytosis was observed (fig 2).

In case 2 also, the superficial layer, composed of hyalinised lamellar collagen bundles, seemed almost intact. But in the deep layer, where the avulsion tear occurred, a granulation tissue response with accumulation of proliferating capillaries, fibroblastic cells, collagen fibres, and a few lymphoid cells, and mild degrees of interstitial oedema and haemorrhage were found. There was a small amount of fibrinous exudate on the torn surface of the ligament. There were no granuloma, vasculitis or neoplastic cells (fig 3).

\section{Discussion}

Many recent reports have shown that the MPFL is present in approximately all knees and is the most important restraint among the medial patellar stabilisers. ${ }^{2-9}$ The MPFL courses from the superior two-thirds of the medial patellar margin superoposteriorly to the medial femoral epicondyle or just distal to the adductor tubercle. ${ }^{2} 10$

The MPFL narrows and thins as it approaches the femoral attachment. ${ }^{2}$ It was expected that the MPFL near the femoral attachment would be weakest structurally, and, if an MPFL injury occurred, the femoral attachment could be the site of injury. In fact, a substantial tear generally occurs near or on the femoral attachment, but did not occur near the patellar attachment in our cases. ${ }^{6}$

An avulsion tear of the MPFL was detected, ${ }^{6}$ which we could not foresee. The superficial fibres of the MPFL pass through the superoposterior portion of the medial femoral epicondyle and extend to the posteromedial capsule. The MPFL seems to have no bony attachment near the medial femoral epicondyle and directly connects to the posteromedial capsule. However, the undersurface of the MPFL at the superoposterior portion of the medial femoral epicondyle is firmly anchored to the bone. We termed this structural characteristic single-point fixation. ${ }^{26}$ Although the specific structure of the femoral attachment is a morphologically and anatomically weak point, it may be that this structure allows 